\title{
Experimental alteration of DNA methylation affects the phenotypic plasticity of ecologically relevant traits in Arabidopsis thaliana
}

\author{
Oliver Bossdorf • Davide Arcuri • Christina L. Richards • \\ Massimo Pigliucci
}

Received: 17 May 2009/Accepted: 8 December 2009/Published online: 19 March 2010

(C) Springer Science+Business Media B.V. 2010

\begin{abstract}
Heritable phenotypic variation in plants can be caused not only by underlying genetic differences, but also by variation in epigenetic modifications such as DNA methylation. However, we still know very little about how relevant such epigenetic variation is to the ecology and evolution of natural populations. We conducted a greenhouse experiment in which we treated a set of natural genotypes of Arabidopsis thaliana with the demethylating agent 5-azacytidine and examined the consequences of this treatment for plant traits and their phenotypic plasticity. Experimental demethylation strongly reduced the growth and fitness of plants and delayed their flowering, but the degree of this response varied significantly among genotypes. Differences in genotypes' responses to demethylation were only weakly related to their genetic relatedness, which is consistent with the idea that natural epigenetic variation is independent of genetic variation. Demethylation also altered patterns of phenotypic plasticity, as well as the amount of phenotypic variation observed among plant individuals and genotype means. We have demonstrated that epigenetic variation can have a dramatic impact on ecologically important plant traits and their variability, as well as on the fitness of plants and their ecological interactions. Epigenetic variation may thus be an overlooked factor in the evolutionary ecology of plant populations.
\end{abstract}

Electronic supplementary material The online version of this article (doi:10.1007/s10682-010-9372-7) contains supplementary material, which is available to authorized users.

O. Bossdorf $(\bowtie)$

Institute of Plant Sciences, University of Bern, 3013 Bern, Switzerland

e-mail: bossdorf@ips.unibe.ch

D. Arcuri

Dipartimento di Biotecnologie (Bio.M.A.A.), Università Mediterranea di Reggio Calabria, 89061 Reggio Calabria, Italy

C. L. Richards

Department of Integrative Biology, University of South Florida, Tampa, FL 33620, USA

M. Pigliucci

Department of Philosophy, City University of New York-Lehman College, Bronx, NY 10468, USA 
Keywords Arabidopsis thaliana $\cdot$ 5-azacytidine · DNA methylation ·

Epigenetics $\cdot$ Natural variation $\cdot$ Phenotypic plasticity

\section{Introduction}

One of the established principles of biology is that evolution by natural selection requires heritable variation, and that the only source of such variation in natural populations is genetic. Therefore, the ability of plant populations to adapt to local habitat conditions or environmental change critically depends on the presence of genetic variation (Endler 1986; Falconer and Mackay 1996; Linhart and Grant 1996); without genetic variation, evolutionary change and adaptation are impossible. However, there is now increasing evidence that, even in the absence of genetic variation, phenotypic variation can be caused by variation in epigenetic modifications of the genome.

The term epigenetics generally refers to a set of molecular processes, including DNA methylation, histone modification, and RNA interference, that can alter gene function and, ultimately, the phenotype without changes in DNA sequence (Grant-Downton and Dickinson 2005; Berger 2007; Bird 2007). The best described of these mechanisms is DNA hypermethylation, where methylation of the $5^{\prime}$-carbon of the cytosine aromatic ring results in transcriptional silencing. Cytosine methylation is also strongly tied to the modification of histones and the condensation of chromatin, and is an important factor in the regulation of gene expression such as the silencing of duplicate genes after polyploid formation (Liu and Wendel 2003; Osborn et al. 2003; Chen 2007). Recent research has shown that there is natural variation in epigenetic modifications, very similar to that in DNA sequence, in many different plant species (e.g. Cervera et al. 2002; Riddle and Richards 2002; Keyte et al. 2006; Vaughn et al. 2007; Zhang et al. 2008), and that at least some of this variation is heritable and independent of genetic variation, and therefore potentially subject to evolution by natural selection (Kalisz and Purugganan 2004; Jablonka and Lamb 2005; Rapp and Wendel 2005; Grant-Downton and Dickinson 2006; Richards 2006; Johannes et al. 2008).

The study of epigenetic inheritance in natural populations is at an early stage. While it is possible that it will eventually significantly improve our understanding of the mechanisms underlying phenotypic variation and the responses of organisms to environmental change, currently there is a dearth of research that has addressed the causes and consequences of epigenetic variation in an evolutionary ecological context. In order to understand the relevance of heritable epigenetic variation, we need to: (1) investigate how much of it is present in natural populations; (2) verify whether epigenetic variation is at least partly independent of genetic variation; and in particular (3) study what sort of significant effects does epigenetic inheritance have on ecologically important traits, on fitness, and on ecological interactions. Here, we have attempted to contribute in particular to the third task.

A clear-cut demonstration of the ecological relevance of epigenetic variation is not a trivial undertaking because of its co-occurrence with genetic variation. To be able to unequivocally attribute phenotypic effects to underlying epigenetic variation, we must, by experimental or statistical means, discern genetic from epigenetic effects (Bossdorf et al. 2008; Johannes et al. 2008). However, the simultaneous analysis of the phenotypic effects of interrelated genetic and epigenetic variation, and their interactions, constitutes a significant methodological and statistical challenge (Johannes et al. 2008). 
Previous studies that convincingly demonstrated phenotypic effects of epigenetic variation often utilized natural or artificial epimutations that allowed the comparison of different epigenotypes of the same genotype (e.g., Cubas et al. 1999). A related technique is to use the demethylating agent 5-azacytidine (5-azaC), a chemical that is incorporated into DNA during replication and thereby inhibits the enzyme methyltransferase and causes partial demethylation of the DNA (e.g., Jones 1985; Burn et al. 1993; Fieldes and Amyot 1999a; Tatra et al. 2000). The obvious advantage of such experimental epimutagenesis is that it allows researchers to create different epigenetic variants of the same genotypes. Because this can be done for many genotypes, it allows us to generalize across different genetic backgrounds, and it is much more amenable to controlled, multi-factorial experiments. The method is somewhat crude, but it can be a valuable first step in exploring the potential phenotypic consequences of epigenetic variation.

Here, we have used 5-azaC to manipulate DNA methylation in a set of natural genotypes of Arabidopsis thaliana and to examine the consequences of these modifications for plant traits and their phenotypic plasticity. Specifically, we have asked the following questions: (1) How does experimental alteration of DNA methylation affect the means and variances of ecologically important phenotypic traits in A. thaliana? (2) Does experimental demethylation alter the degree of phenotypic plasticity of plants in response to different nutrient levels? (3) Do these responses to 5-azaC vary among different Arabidopsis genotypes? (4) If yes, are genotypic differences in demethylation responses related to the genetic distances among genotypes?

\section{Materials and methods}

\section{Plant material}

Arabidopsis thaliana (L.) Heynh. (Brassicaceae) is a small annual weed in the mustard family (Brassicaceae). It is a predominantly selfing, ruderal species native to Eurasia but now widely naturalized in the USA and elsewhere. A. thaliana has long been a model species for plant genetics and molecular biology (Meyerowitz and Somerville 2002). However, because of the growing body of knowledge about its physiology, development and molecular biology, the logistic advantages it affords, and the availability of a broad array of molecular tools, the species is now also frequently used as a model for addressing basic questions at the interface of ecology and evolutionary biology (Pigliucci 1998; Mitchell-Olds 2001; Koornneef et al. 2004). In this study, we used a total of 22 genotypes of A. thaliana: 20 genotypes from natural populations, representing the species' widest geographical range (Table S1), and the two standard laboratory lines Landsberg erecta (Ler-2) and Columbia (Col). The seeds were obtained from the Arabidopsis Stock Center (TAIR; www.arabidopsis.org) where these genotypes, originally collected in the field, have been propagated and maintained under uniform conditions for several generations.

\section{Experiment}

We conducted an experiment in which the 22 Arabidopsis genotypes were subjected to a factorial combination of nutrient addition and experimental demethylation treatments. In January 2006, all seeds were cold-stratified in the dark at $4^{\circ} \mathrm{C}$ for 1 week. For each genotype, we used two petri dishes, one in which the filter paper was moistened with $1.4 \mathrm{ml}$ water, and one in which it was moistened with $1.4 \mathrm{ml}$ of a $50 \mu \mathrm{mol} 5$-azacytidine 
(5-azaC; Sigma Scientific, St. Louis, MO) solution. After this stratification period, we transferred the petri dishes to an unheated greenhouse for another week under natural light conditions until most seeds had germinated. The seedlings were transplanted into 72-cell planting trays filled with a 50:50 mix of sand and vermiculite. The experiment followed a split plot design with trays as plots, 5-azaC and nutrient treatments as whole plot factors, and plant genotype as subplot factor. Low and high nutrient levels were created by adding either one or eight pellets of a slow-release fertilizer (15-9-12 Osmocote Plus 8-9 M, Scotts Miracle-Gro, Marysville, $\mathrm{OH}$ ) to each cell in a planting tray. For each treatment combination, there were three planting trays, each with three replicates per genotype. Thus, there were 12 replicates of each genotype in each treatment combination, and a total of 1056 plants in the experiment. The trays were arranged randomly on two greenhouse benches and watered as needed throughout the experiment. After approximately 45 days, the first genotypes started flowering. Each plant was harvested at fruit maturity, 30 days after first flowering. For each plant, we recorded (1) time to flowering, as the number of days between the start of the greenhouse experiment and the first appearance of a white petal, (2) rosette diameter to the nearest millimeter as a measure of plant size at the time of flowering, (3) final plant height to the nearest millimeter, (4) the total number of basal and lateral branches, (5) the total number of siliques, and (6) the final aboveground biomass in milligrams after drying at $60^{\circ} \mathrm{C}$ for $24 \mathrm{~h}$.

\section{Statistical analyses}

We used nested analysis of variance to test for the effects of demethylation, nutrient availability, genotype, and their interactions on each of the response variables. The effects of 5-azaC, nutrients and their interaction were tested at the plot level, using the tray mean squares as error term, whereas the genotype effects and their interactions were tested at the level of plant individuals, i.e. against the residual. To improve normality and homoscedasticity of the data, flowering time and plant height were square-root transformed, and all other variables were log-transformed prior to the analyses. In addition, we ran a generalized linear model with binomial error and logit link to test for the effects of treatments, genotypes and their interactions on plant mortality. We used the $\chi^{2}$ deviances to calculate variance ratios that were approximately $F$-distributed (McCullagh and Nelder 1989). All analyses were done in JMP 6.0.3 (SAS Institute, Cary, NC).

To examine the effects of 5-azaC on phenotypic variation, we calculated coefficients of variation for each phenotypic trait in the control versus 5-azaC treatment, and we used the asymptotic test statistic by Miller (1991) to test for differences between these CVs. We ran these tests both for the raw phenotypic variation among plant individuals, and for the variation among genotype means, an estimate of quantitative genetic variation. As data transformations equalize variances, the $\mathrm{CVs}$ were calculated from untransformed data. The results of the genotype-level analyses did not qualitatively differ when CVs were calculated from raw genotypic means instead of least square means; we therefore present only the results for comparisons of CVs based on raw data.

Finally, we examined whether differences between genotypes in their phenotypic responses to 5-azaC were related to their genetic distance. We used data on 149 singlenucelotide polymorphisms (SNPs) provided by Justin Borevitz (available at http://naturalvariation.org/hapmap) and the dnadist program in PHYLIP (Felsenstein 2005) to construct a genetic distance matrix of the 22 genotypes. Next, we calculated seven Euclidean distance matrices based on the average percent phenotypic change of each genotype in response to 5-azaC treatment: one matrix for each of the six traits, and one in 
which the changes in all traits were considered together. As the degree of change differed greatly among traits, the response data were standardized prior to distance calculations. We used Mantel tests to assess correlations between genetic and phenotypic distances. Distance calculations and Mantel tests were done using the ecodist package (Goslee and Urban 2007) in R (R Development Core Team 2007).

\section{Results}

Out of the 1056 individuals planted, 467 reached maturity. Plants treated with 5-azaC had more than $40 \%$ higher rate of mortality than plants that were not treated (Table 1). Analysis of deviance indicated that mortality was also significantly affected by plant genotype and the 5-azaC by genotype interaction (Table 2). However, the full statistical model explained only $15 \%$ of the total variation, so most of the mortality, which was mostly likely caused by temperature stress in the greenhouse, was unrelated to any of the experimental factors.

Table 1 Phenotypic differences between Arabidopsis thaliana plants germinated with or without 5-azacytidine

\begin{tabular}{lrcc}
\hline & \multicolumn{1}{c}{ Control } & 5-azaC & \% Change \\
\hline Mortality & $45.83 \%$ & $65.72 \%$ & +43.4 \\
Flowering time (d) & $50.19( \pm 0.78)$ & $56.73( \pm 0.63)$ & +13.0 \\
Size at flowering (cm) & $1.91( \pm 0.08)$ & $1.29( \pm 0.08)$ & -32.2 \\
Plant height (cm) & $15.77( \pm 0.50)$ & $11.89( \pm 0.56)$ & -24.6 \\
Branch number & $5.39( \pm 0.25)$ & $3.77( \pm 0.27)$ & -30.0 \\
Biomass (mg) & $49.96( \pm 3.90)$ & $24.05( \pm 3.07)$ & -51.9 \\
Fruit number & $59.91( \pm 3.58)$ & $37.71( \pm 4.58)$ & -37.1 \\
\hline
\end{tabular}

Size at flowering $=$ rosette diameter

The values are means \pm standard errors from the raw data

Table 2 Summary of analysis of deviance of plant mortality

\begin{tabular}{lccc}
\hline Source & DF & Mean deviance & Quasi-F \\
\hline Plot level & & & \\
5-Azacytidine & 1 & 46.78 & $30.36^{* * * *}$ \\
Nutrients & 1 & 3.83 & 2.49 \\
$\mathrm{~A} \times \mathrm{N}$ & 1 & 2.99 & 1.94 \\
Tray & 12 & 1.54 & 1.19 \\
Plant level & & & \\
Genotype & 21 & 3.09 & $2.38^{* * *}$ \\
$\mathrm{~A} \times \mathrm{G}$ & 21 & 2.93 & $2.26^{* *}$ \\
$\mathrm{~N} \times \mathrm{G}$ & 21 & 0.54 & 0.41 \\
$\mathrm{~A} \times \mathrm{N} \times \mathrm{G}$ & 21 & 0.53 & 0.41 \\
Residual & 956 & 1.30 & \\
\hline
\end{tabular}

$D F$ degrees of freedom. ** $P<0.01$, *** $P<0.001$ 


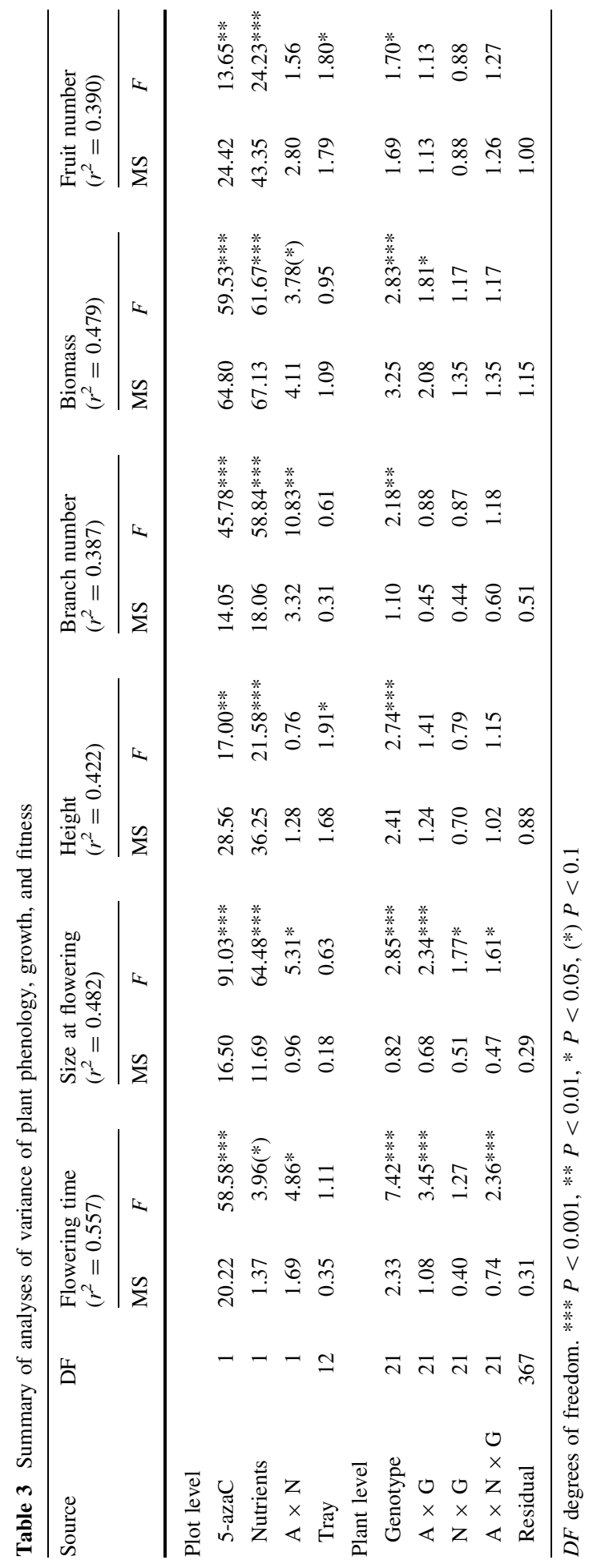


Experimental demethylation by 5-azaC strongly affected all of measured traits (Table 1). On average, plants treated with 5-azaC flowered over 7 days later, and were significantly smaller at the time of flowering than control plants (Table 1). Moreover, plants treated with 5-azaC suffered a significant reduction in plant height and biomass, as well as the numbers of branches and fruits produced (Table 1).

There was significant genetic variation in the degree to which 5-azaC affected flowering time, size at flowering, and plant biomass (Table 3; Fig. 1). While in most Arabidopsis genotypes, treatment with 5-azaC (averaged across both nutrient treatments) clearly delayed flowering, the opposite was true for at least four genotypes (Fig. 1). Similarly, size at flowering and total biomass were usually dramatically reduced by 5 -azaC, but there were a few genotypes that maintained or even increased their size in response to demethylation.

Treatment with 5-azaC generally altered plant responses to nutrient addition (Table 3; Fig. 2). On average, demethylated plants that grew at high nutrient levels flowered 4.4 days later than demethylated plants at low nutrient levels, while in control (i.e., nondemethylated) plants there was a tendency for nutrients to have the opposite effect on flowering time (Fig. 2a). In addition, demethylated plants showed a significantly weaker response to nutrient addition with regard to plant size at flowering and average branch numbers (Fig. 2b/c). There was a significant 3-way interaction between the effects of 5 -azacytidine, nutrients and genotype on flowering time, indicating that the degree to which 5-AzaC altered the plasticity of this trait to nutrient additions varied among Arabidopsis genotypes (Fig. 3). For instance, while control plants of the Bologna (Bl-1) and Coimbra (Co-1) accessions showed identical phenological responses to nutrient additions, treatment with 5-azaC induced a strong delay of flowering in response to nutrients in the Spanish population, but did not change the response of the Italian population (Fig. 3).

Experimental treatment with 5-azaC significantly increased the phenotypic variability among individuals in five out of six traits (Fig. 4a). The strongest effect was on reproduction, where treatment with 5 -azaC increased the coefficient of variation by almost $60 \%$. The picture was slightly different when we examined genotypic means (Fig. 4b). Overall coefficients were much lower, and while treatment with 5-azaC more than doubled the estimated genotypic variation in fruit production and increased genotypic variation in branch number, it did not significantly affect variability in other traits such as plant height and biomass, and it even decreased the estimated genotypic variation in flowering time.

When constructing genetic distance matrices, the genotypes Di-2 and Ler-2 turned out to be genetically identical, based on the SNP data used. The distance between these two genotypes constituted an extreme outlier in the matrix correlations, and we therefore omitted it from these analyses. When all phenotypic traits were considered together, there
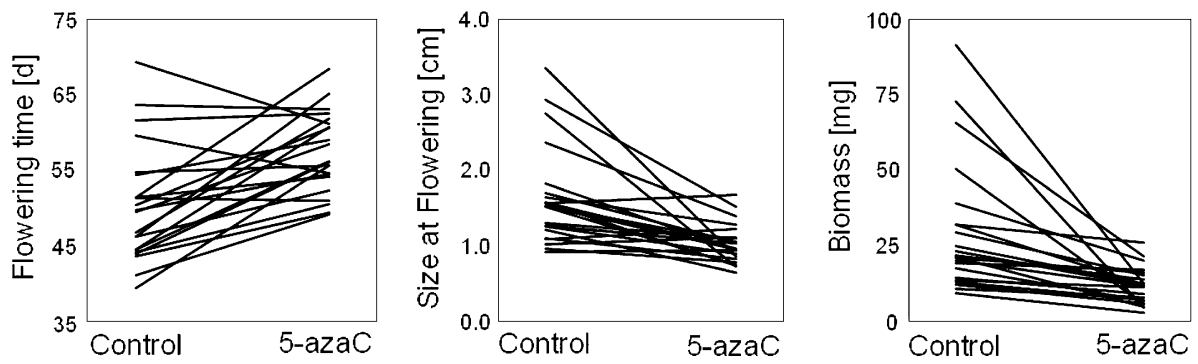

Fig. 1 The variation among 22 genotypes of Arabidopsis thaliana in their phenotypic responses to experimental demethlyation. Each line represents a different genotype 

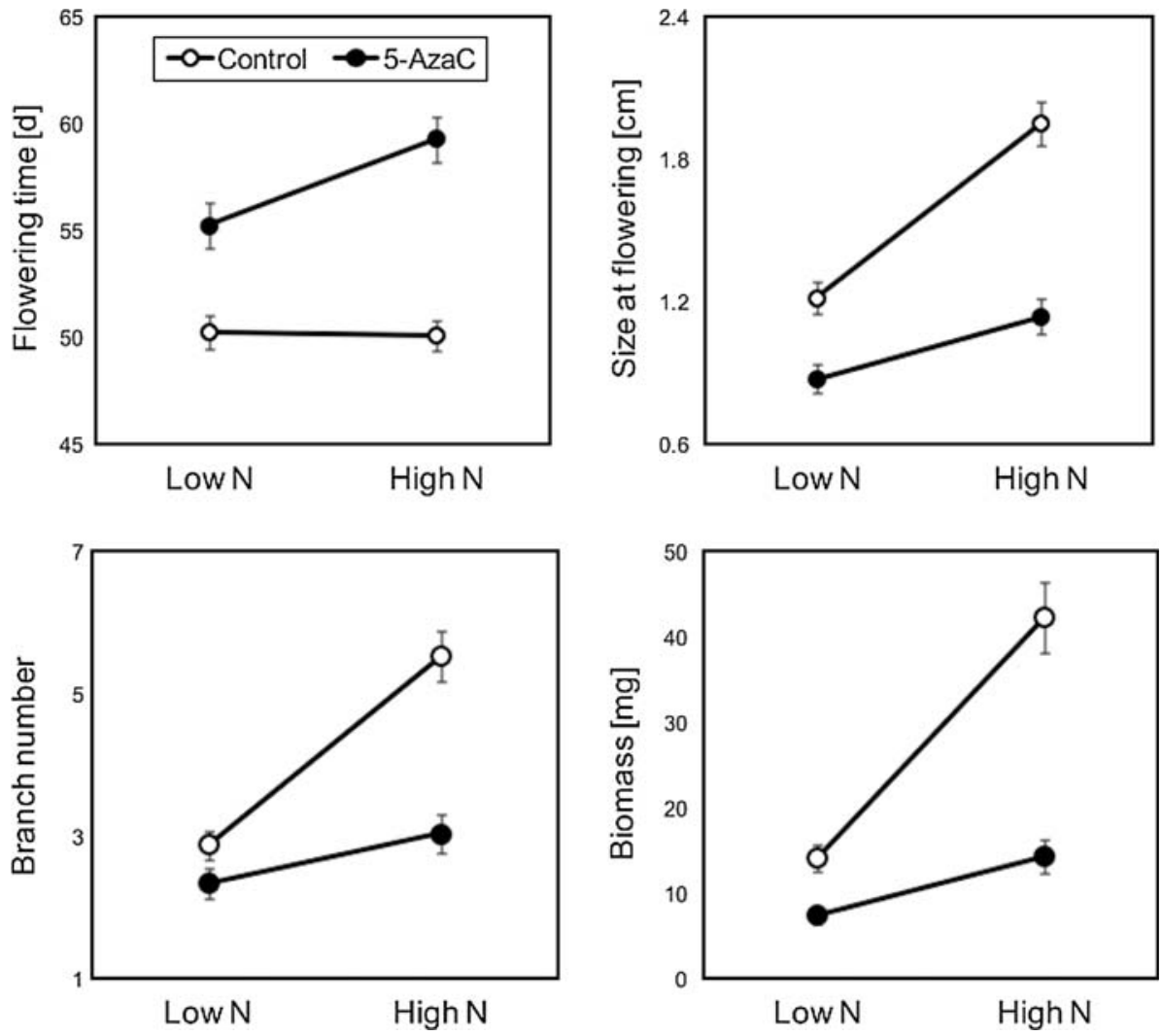

Fig. 2 The effect of experimental demethylation through 5-azacytidine on the average phenotypic plasticity of 22 genotypes of Arabidopsis thaliana in response to increased nutrient availability

Fig. 3 Genotypic variation in the effects of experimental demethylation on plant phenotypic plasticity. The reaction norms illustrate how treatment with 5-azacytidine alters the phenological response to nutrient additions in 22 genotypes of Arabidopsis thaliana. Two genotypes with contrasting responses are highlighted in bold

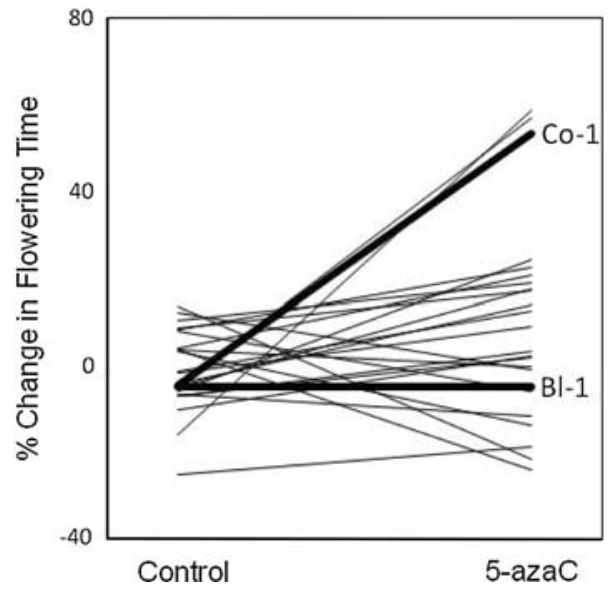

was only a marginally significant correlation between the genetic distance of genotypes and the dissimilarity of their phenotypic responses to 5-azaC treatment (Table 4). When traits were considered separately, there was a significant correlation between genetic 

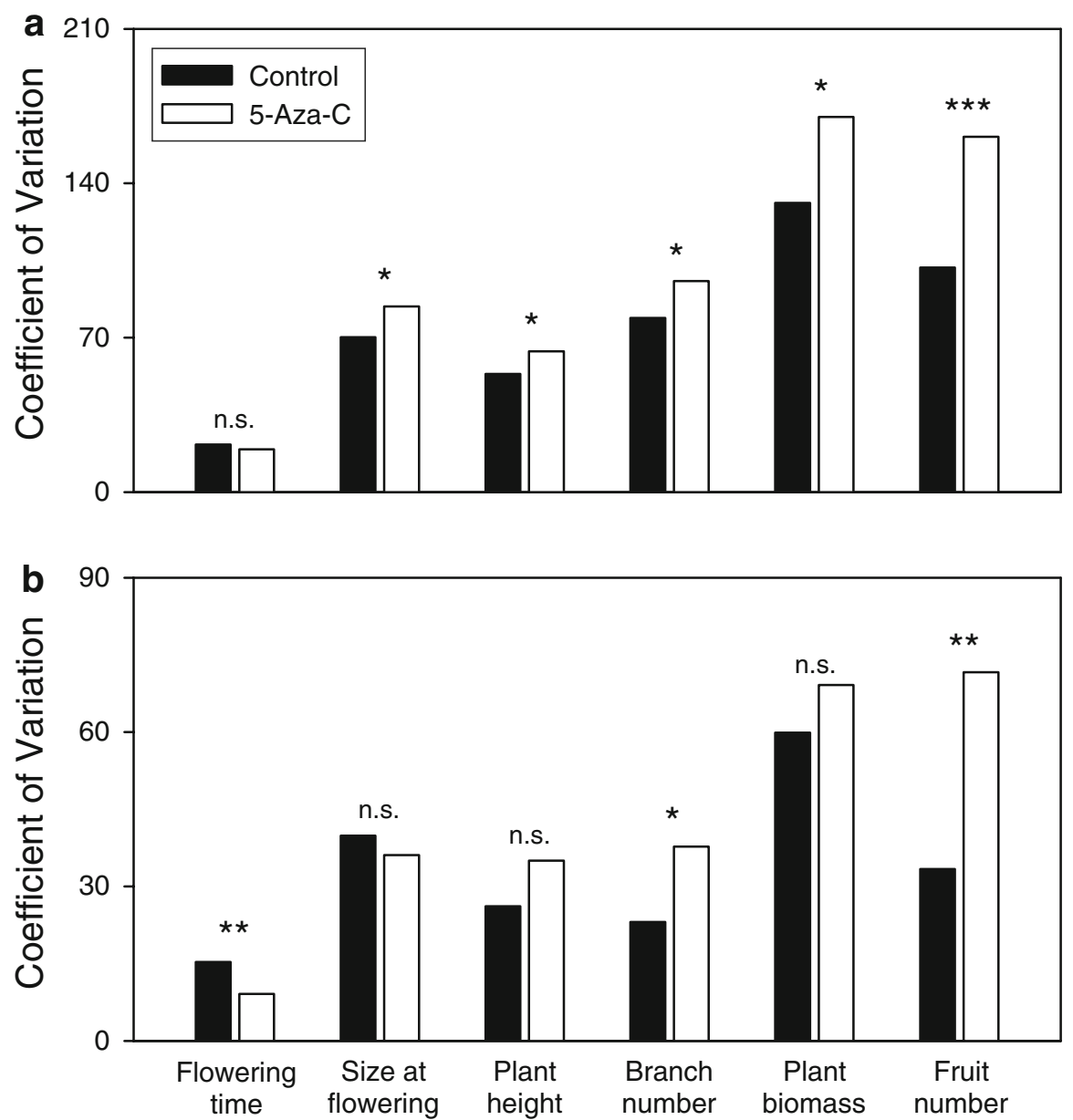

Fig. 4 The effects of experimental demethylation through 5-azacytidine on phenotypic variability among 22 genotypes of Arabidopsis thaliana. a Coefficient of variation among plant individuals. b Coefficient of variation of genotypic means. *** $P<0.001$, ** $P<0.01, * P<0.05$, n.s. $=$ not significant

Table 4 Mantel correlation tests between the genetic distances among 22 Arabidopsis genotypes and the dissimilarity of their phenotypic responses to 5-azacytidine treatment

\begin{tabular}{lcc}
\hline Phenotypic response in terms of... & Correlation & Significance \\
\hline ALL TRAITS & 0.1421 & 0.0716 \\
Flowering time & -0.0339 & 0.3364 \\
Size at flowering & 0.1417 & 0.0549 \\
Height & 0.1906 & 0.0158 \\
Branch number & -0.0328 & 0.3603 \\
Biomass & 0.2030 & 0.0057 \\
Fruit number & 0.1147 & 0.1160 \\
\hline
\end{tabular}

Significance levels are based on 100,000 permutations 
distance and the height and biomass responses to 5-azaC, whereas correlations were nonsignificant for all other traits (Table 4). However, none of the significant correlations explained more than $4 \%$ of the variation in the data. These results were very consistent and did not change qualitatively when we re-ran the analyses using Jukes-Cantor genetic distances rather than simple distances based on the fraction of identical sites. We therefore present only the results for simple genetic distances in Table 4.

\section{Discussion}

Although there is increasing evidence of heritable epigenetic variation in plants (e.g. Cervera et al. 2002; Riddle and Richards 2002; Keyte et al. 2006; Vaughn et al. 2007; Zhang et al. 2008), few connections have been made so far between epigenetic variation and the variability and phenotypic plasticity of ecologically important plant traits (Bossdorf et al. 2008). To improve our understanding of the ecological relevance of epigenetic variation, we conducted a greenhouse experiment in which we used 5-azacytidine to manipulate DNA methylation in a set of natural genotypes of Arabidopsis thaliana.

In our study, we did not use molecular methods to verify demethylation at the molecular level. However, many previous studies have demonstrated this effect of 5-azaC (e.g., Burn et al. 1993; Tatra et al. 2000; Fieldes et al. 2005), and we think it is reasonable to assume that reduced DNA methylation was the main cause of the observed phenotypic changes in 5-azaC-treated plants. One alternative explanation we cannot rule out is that demethylation induced mobilization of transposable elements (Johannes et al. 2009), and that at least some of the observed phenotypic changes results from such induced DNA sequence changes. However, little is known about the frequency and phenotypic effects of such transpositions, and it is very difficult to evaluate the likelihood of this alternative.

We found that experimental alteration of DNA methylation strongly affected the growth, fitness and phenology of A. thaliana. There were large differences between demethylated and control plants of the same genotype. A few other studies have also demonstrated effects of demethylation through 5-azaC on ecologically important traits. Burn et al. (1993) showed that treatment of A. thaliana and Thlaspi arvense with 5-azaC significantly altered plant flowering times. In a series of experiments, Fieldes and colleagues (Fieldes 1994; Fieldes and Amyot 1999b; Fieldes et al. 2005) found that 5-azaC affected the growth, fitness and phenology of Linum usitatissimum. These studies, and the one presented here, show that epigenetic variation, independently of DNA variation, can cause major shifts in plant phenotypes.

In most genotypes, demethylation caused a decrease in overall plant performance. This general effect of demethylation or treatment with 5-azaC has been found by several previous studies (e.g., Burn et al. 1993; Fieldes 1994; Finnegan et al. 1996). Demethylation resulted in a significant delay of plant flowering: on average, 5-azaC-treated plants flowered 6.5 days later than control plants. This is in apparent contrast with several previous studies that found 5-azaC or demethylation to induce early flowering in Arabidopsis (e.g., Burn et al. 1993; Finnegan et al. 1996, 1998) and other plant species (e.g., Fieldes and Amyot 1999b; Kondo et al. 2007). However, recent research has shown that the transition to flowering in Arabidopsis is a complex developmental process that involves both methylation and demethylation of specific genes, and that, depending on the genetic background, hypomethylation may either cause early or late flowering, or have no effect on flowering time at all (Genger et al. 2003). Previous studies that found demethylation to induce early flowering in Arabidopsis had often been using the artificial Landsberg erecta 
(Ler) line or Ler-based mutants. In our study, however, only two out of 22 Arabidopsis ecotypes (Abd-0, Oy-0) flowered earlier when treated with 5-azaC, whereas in 13 ecotypes flowering time was prolonged by over $5 \%$. This suggests that the Ler ecotype may not be representative of natural variation in Arabidopsis thaliana.

There was significant variation among Arabidopsis ecotypes in their responses to experimental demethylation. However, these ecotypic differences in demethylation responses were only weakly related to the genetic distances between genotypes, which is consistent with the idea of natural epigenetic variation being independent of the corresponding genetic variation (Richards 2006; Bossdorf et al. 2008). Alternatively, ecotypic differences in the effects of 5-azaC could result from some kind of mechanical or physiological differences between ecotypes that affect the absorption, transport and/or incorporation of 5-azaC, but are at the same time uncorrelated to DNA sequence variation, or they could be-at least theoretically-due to ecotype-specific transposon activation. Both alternatives are rather speculative, and we think that epigenetic variation is indeed the most parsimonious explanation, particularly since its existence in Arabidopsis thaliana has already been shown several times (e.g., Cervera et al. 2002; Riddle and Richards 2002; Vaughn et al. 2007).

Treatment with 5-azaC increased phenotypic variability in most traits, which suggests that 5-azaC may not be a very precise demethylation agent and it may therefore not only reduce overall levels of methylation but also increase variation in methylation. Our result is consistent with a study of Fieldes and Amyot (1999b) who found such effects of 5-azaC were still present even in the fourth generation of untreated offspring. However, treatment with 5-azaC not only altered overall phenotypic variability but also the apparent variability of genotypic means of some traits. This has immediate implications for our understanding of evolutionary processes, as measures of genotypic variation are commonly used for estimating broad-sense heritability, one of the key parameters for predicting microevolution (e.g. Falconer and Mackay 1996). Thus, epigenetic variation can alter estimates of evolutionary relevant phenotypic variation and of evolutionary potential.

Altering DNA methylation in Arabidopsis not only changed the means and variability of ecologically important traits, but also their phenotypic plasticity. Demethylation decreased the overall plasticity of plants to nutrient addition with regard to several morphological parameters, and it substantially increased the plasticity of flowering time. We are only aware of one previous study that experimentally tested for the effects of variation in DNA methylation on plant phenotypic plasticity: Tatra et al. (2000) subjected two ecotypes of Stellaria longipes to a factorial combination of light and 5-azaC and found that the experimental demethylation significantly altered plants' response to light. In our study, as well as in the one by Tatra et al. (2000), there were significant differences between different genotypes in the way that demethylation affected phenotypic plasticity (significant three-way interaction between genotype, demethylation, and environmental treatment), which suggests that genotype and epigenotype may interact in complex ways to determine phenotypic plasticity.

In summary, epigenetic changes such as reduced DNA methylation can have a dramatic impact on the mean, variability and plasticity of ecologically important plant traits. Epigenetic variation may thus be an overlooked factor in the evolutionary ecology of plant populations.

Acknowledgments This work was partially supported by the NSF IOB-0450240, the Research Foundation of the State University of New York and New York SEA Grant. We are grateful to Justin Borevitz for providing the SNP data, and to Stefan Michalski for his help with the genetic distance matrices. 


\section{References}

Berger SL (2007) The complex language of chromatin regulation during transcription. Nature 447:407-412

Bird A (2007) Perceptions of epigenetics. Nature 447:396-398

Bossdorf O, Richards CL, Pigliucci M (2008) Epigenetics for ecologists. Ecol Lett 11:106-115

Burn JE, Bagnall DJ, Metzger JD, Dennis ES, Peacock WJ (1993) DNA methylation, vernalization, and the initiation of flowering. Proc Natl Acad Sci USA 90:287-291

Cervera MT, Ruiz-Garcia L, Martinez-Zapater JM (2002) Analysis of DNA methylation in Arabidopsis thaliana based on methylation-sensitive AFLP markers. Mol Genet Genom 268:543-552

Chen ZJ (2007) Genetic and epigenetic mechanisms for gene expression and phenotypic variation in plant polyploids. Annu Rev Plant Biol 58:377-406

Cubas P, Vincent C, Coen E (1999) An epigenetic mutation responsible for natural variation in floral symmetry. Nature 401:157-161

Endler JA (1986) Natural selection in the wild. Princeton University Press, Princeton

Falconer DS, Mackay TFC (1996) Introduction to quantitative genetics. Pearson, Harlow

Felsenstein J (2005) PHYLIP version 3.6. Distributed by the author, Department of Genome Sciences, University of Washington

Fieldes MA (1994) Heritable effects of 5-azacytidine treatments on the growth and development of flax (Linum usitatissimum) genotrophs and genotypes. Genome 37:1-11

Fieldes MA, Amyot LM (1999a) Evaluating the potential of using 5-Azacytidine as an epimutagen. Can J Bot 77:1617-1622

Fieldes MA, Amyot LM (1999b) Epigenetic control of the early flowering in flax lines induced by 5-Azacytidine applied to germinating seeds. J Hered 90:199-206

Fieldes MA, Schaeffer SM, Krech MJ, Brown JCL (2005) DNA hypomethylation in 5-azacytidine-induced early-flowering lines of flax. Theor Appl Genet 111:136-149

Finnegan EJ, Peacock WJ, Dennis ES (1996) Reduced DNA methylation in Arabidopsis thaliana results in abnormal plant development. Proc Natl Acad Sci USA 93:8449-8454

Finnegan EJ, Genger RK, Kovac K, Peacock WJ, Dennis ES (1998) DNA methylation and the promotion of flowering by vernalization. Proc Natl Acad Sci USA 95:5824-5829

Genger RK, Peacock WJ, Dennis ES, Finnegan EJ (2003) Opposing effects of reduced DNA methylation on flowering time in Arabidopsis thaliana. Planta 216:461-466

Goslee SC, Urban DL (2007) The ecodist package for dissimilarity-based analysis of ecological data. J Stat Softw 22:7 (www.jstatsoft.org)

Grant-Downton RT, Dickinson HG (2005) Epigenetics and its implications for plant biology. 1. The epigenetic network in plants. Ann Bot 96:1143-1164

Grant-Downton RT, Dickinson HG (2006) Epigenetics and its implications for plant biology. 2. The 'epigenetic epiphany': epigenetics, evolution and beyond. Ann Bot 97:11-27

Jablonka E, Lamb MJ (2005) Evolution in Four Dimensions. MIT Press, Cambridge

Johannes F, Colot V, Jansen RC (2008) Epigenome dynamics: a quantitative genetics perspective. Nature Rev Genet 9:883-890

Johannes F, Porcher E, Teixeira FK, Saliba-Colombani V, Simon M, Agier N, Bulski A, Albuisson J, Heredia F, Audigier P, Bouchez D, Dillmann C, Guerche P, Hospital F, Colot V (2009) Assessing the impact of transgenerational epigenetic variation on complex traits. PLoS Genet 5:e1000530

Jones PA (1985) Altering gene expression with 5-Azacytidine. Cell 40:485-486

Kalisz S, Purugganan MD (2004) Epialleles via DNA methylation: consequences for plant evolution. Trends Ecol Evol 19:309-314

Keyte AL, Percifield R, Liu B, Wendel JF (2006) Infraspecific DNA methylation polymorphism in cotton (Gossypium hirsutum L.). J Hered 97:444-450

Kondo H, Miura T, Wada KC, Takeno K (2007) Induction of flowering by 5-azacytidine in some plant species: relationship between the stability of photoperiodically induced flowering and flower-inducing effect of DNA demethylation. Physiol Plant 131:462-469

Koornneef M, Alonso-Blanco C, Vreugdenhil D (2004) Naturally occurring genetic variation in Arabidopsis thaliana. Annu Rev Plant Biol 55:141-172

Linhart YB, Grant MC (1996) Evolutionary significance of local differentiation in plants. Annu Rev Ecol Syst 27:237-277

Liu B, Wendel JF (2003) Epigenetic phenomena and the evolution of plant allopolyploids. Mol Phylogenet Evol 29:365-379

McCullagh P, Nelder JA (1989) Generalized linear models. CRC Press, Boca Raton 
Meyerowitz EM, Somerville CR (2002) The Arabidopsis book. American Society of Plant Biologists, Rockville. Available from http://www.aspb.org/publications/arabidopsis/

Miller GE (1991) Asymptotic test statistics for coefficients of variation. Commun Stat Theor M 20: $3351-3363$

Mitchell-Olds T (2001) Arabidopsis thaliana and its wild relatives: a model system for ecology and evolution. Trends Ecol Evol 16:693-700

Osborn TC, Pires JC, Birchler JA, Auger DL, Chen ZJ, Lee HS, Comai L, Madlung A, Doerge RW, Colot V, Martienssen RA (2003) Understanding mechanisms of novel gene expression in polyploids. Trends Genet 19:141-147

Pigliucci M (1998) Ecological and evolutionary genetics of Arabidopsis. Trends Plant Sci 3:485-489

Rapp RA, Wendel J (2005) Epigenetics and plant evolution. New Phytol 168:81-91

Richards EJ (2006) Inherited epigenetic variation-revisiting soft inheritance. Nature Rev Genet 7:395-401

Riddle NC, Richards EJ (2002) The control of natural variation in cytosine methylation in Arabidopsis. Genetics 162:355-363

Tatra GS, Miranda J, Chinnappa CC, Reid DM (2000) Effect of light quality and 5-azacytidine on genomic methylation and stem elongation in two ecotypes of Stellaria longipes. Physiol Plant 109:313-321

R Development Core Team (2007) R: A language and environment for statistical computing. R Foundation for Statistical Computing, Vienna. Available from http://R-project.org

Vaughn MW, Tanurdzic M, Lippman Z, Jiang H, Carrasquillo R, Rabinowicz PD, Dedhia N, McCombie WR, Agier N, Bulski A, Colot V, Doerge RW, Martienssen RA (2007) Epigenetic natural variation in Arabidopsis thaliana. PLoS Biol 5:e174

Zhang X, Shiu S, Cal A, Borevitz JO (2008) Global analysis of genetic, epigenetic and transcriptional polymorphisms in Arabidopsis thaliana using whole genome tiling arrays. PLoS Genet 4:e1000032 